\title{
Gilles Ferréol (dir.), Tourisme et patrimoine
}

coll. "Proximités Sociologie", EME \& Intercommunications, 2010, 298 p.

\section{Michel Raspaud}

\section{OpenEdition}

\section{Journals}

Édition électronique

URL : http://journals.openedition.org/tourisme/479

DOI : $10.4000 /$ tourisme.479

ISSN : 2492-7503

\section{Éditeur}

Éditions touristiques européennes

\section{Édition imprimée}

Date de publication : 1 décembre 2011

Pagination : 110-111

ISSN : 2109-5671

\section{Référence électronique}

Michel Raspaud, « Gilles Ferréol (dir.), Tourisme et patrimoine », Mondes du Tourisme [En ligne], 4 | 2011, mis en ligne le 30 septembre 2015, consulté le 22 septembre 2020. URL : http://

journals.openedition.org/tourisme/479; DOI : https://doi.org/10.4000/tourisme.479

Ce document a été généré automatiquement le 22 septembre 2020.

\section{$(1) \Theta$}

Mondes du tourisme est mis à disposition selon les termes de la licence Creative Commons Attribution - Pas d'Utilisation Commerciale - Pas de Modification 4.0 International. 


\section{Lecture critique}

\section{Gilles Ferréol (dir.), Tourisme et patrimoine}

coll. "Proximités Sociologie", EME \& Intercommunications, 2010, 298 p.

\section{Michel Raspaud}

\section{RÉFÉRENCE}

Gilles Ferréol (dir.), Tourisme et patrimoine, coll. "Proximités Sociologie”, EME

\& Intercommunications, 2010.

Cet ouvrage rend compte d'un colloque pluridisciplinaire organisé à Confolens (PoitouCharentes) en mars 2010, à l'initiative de l'Arpe (Association régionale pour la promotion de l'ethnologie en Poitou-Charentes) et du laboratoire Culture, sport, société de l'université de Franche-Comté. Il fait suite à une première session de ce genre, tenue à Perpignan deux ans plus tôt. Ce colloque mêle des intervenants restituant des analyses et des expériences multiples, avec des enseignants chercheurs, des doctorants et jeunes docteurs, des responsables associatifs, des élus locaux. Parmi l'ensemble des dix-neuf communications, six sont faites par des non-universitaires stricto sensu, ce qui démontre l'ouverture aux acteurs de terrain du patrimoine. On peut relever la diversité de ces intervenants : une chargée d'étude spécialisée dans l'inventaire du patrimoine, la conservatrice territoriale en chef du patrimoine de Poitou-Charentes, une animatrice du Pays d'art et d'histoire du Confolentais, la directrice adjointe de Via Patrimoine, un enseignant retraité et militant associatif, le responsable du service de l'Inventaire général du patrimoine culturel de la région Poitou-Charentes, et la chargée de mission pour le projet de la Cité du cuir à Lavausseau. L'ouvrage est organisé en deux parties : dans un premier temps, huit textes proposent des éléments de cadrage et de problématisation quant à la question du patrimoine; dans un second temps, onze illustrations et études de cas sont présentées. 
2 Dans la première partie, il est fait référence à Georges Henri Rivière et à ses réflexions et actions en faveur des arts et traditions populaires, avec la question de la complexité de la notion de patrimoine, ses extensions et ramifications possibles, son aspect protéiforme. Michel Valière parle alors de la patrimonialisation comme dynamique sociale, et plus précisément du processus de patrimonialisation lui-même, c'est-à-dire le rapport au patrimoine via les interrogations sur ses "modalités actuelles, sociales et politiques, notamment celles du travail de production symbolique des identités collectives, et plus généralement le rapport au passé et à la culture..." Il souligne que, aujourd'hui, la notion a perdu toute limite sémantique, au point qu'à peu près tout peut faire patrimoine.

3 C'est dans cette logique que s'inscrit Laurent Sébastien Fournier, puisqu'il s'attache aux nouvelles catégories patrimoniales, ce qu'il nomme le "petit patrimoine" : les objets qui le constituent sont en relation directe avec le monde de l'enfance et avec les parcours biographiques des individus qui les possèdent. Il prend comme exemple le petit patrimoine des clubs sportifs mis en exposition (coupures de presse, licences, photographies anciennes, etc., en plus des maillots, coupes...). Pour L. S. Fournier, ce petit patrimoine "permet à des communautés peu dotées sur le plan du grand patrimoine de développer, à leur niveau, une conscience vive de leur singularité". Les autres textes de cette première partie traitent :

- de l'inventaire du patrimoine comme mission de connaissance au service de la valorisation culturelle des territoires (Sonia Servant);

- de la culture et la littérature populaires - roman populaire, mythe, roman policier, récit comme vecteurs de la transmission patrimoniale (Brigitte Munier);

- des sociétés du risque et de la précaution patrimoniale - un espace agricole du Cotentin rendu "archaïque" par les nouvelles normes des politiques agricoles française et européenne (Yves Dupont) ;

- du déficit de candidatures émanant des gens du voyage à l'appel d'offres de l'Unesco 2008-2009 pour un classement au titre de patrimoine culturel immatériel (AnneMarie Mamontoff) ;

- du choix fait par quelques collectivités territoriales de mettre en valeur, pour leur développement touristique, des sites archéologiques relevant du réseau romain des grandes villes, des agglomérations secondaires, des grandes exploitations agricoles et des routes (Véronique Dujardin);

- de l'histoire vivante définie comme "toute re-création et mise en scène d'un événement historique particulier ou d'une manière de faire du temps passé", avec l'exemple du musée des Temps barbares de Marle et son festival d'histoire vivante qui, lors des journées de juin 2009, ont fait 7000 entrées pour un village de 2500 habitants (Audrey Tuaillon-Demésy).

4 La seconde partie de l'ouvrage restitue onze études de cas très variées, pour un certain nombre d'entre elles relatives à la région Poitou-Charentes, mais explorant aussi d'autres zones géographiques françaises, voire d'autres temporalités (plongée dans l'histoire) :

- le Marais poitevin, d'un paysage pittoresque à sa patrimonialisation à travers l'écotourisme (Daniel Bourdu) ;

- les étangs de la Brenne (organisés entre eux en chaînes et voués aux activités halieutiques et cynégétiques, nécessitant une gestion commune afin d'assurer le bon fonctionnement du système), avec l'eau constituant un enjeu écologique, économique mais aussi social et symbolique pour la communauté locale (Karen Julien) 
- le rôle et la mission d'un territoire labellisé Ville ou Pays d'art et d'histoire (Céline Deveza et Laetitia Copin-Merlet) ;

- le tourisme vert et la patrimonialisation des paysages ordinaires du Sud-Vienne en Poitou (Henri Donzaud) ;

- le Jardin de Gabriel à Nantillé, Charente-Maritime, site touristique en voie de patrimonialisation (Fabrice Bonnifait);

- le projet d'une Cité du cuir sur une commune de 800 habitants du département de la Vienne, Lavausseau (Catherine Durepaire);

- le regard sur un moment historique, celui du tournant $\mathrm{XIX}^{\mathrm{e}}-\mathrm{XX}^{\mathrm{e}}$ siècles et le tourisme et le patrimoine canotiers à Besançon : les activités organisées par les membres "sélects" de la Société nautique de Besançon sont tournées vers la cité et le touriste étranger jusqu'à la dissolution de cette dernière en 1900 ; ensuite, la nouvelle association du Sport nautique s'oriente plus vers les classes moyennes et évolue vers des animations et un tourisme pour ses propres adhérents (Christian Vivier et Jean-Nicolas Renaud) ;

- la Transjurassienne, la plus célèbre course populaire de ski de fond de France (76 km entre Lamoura, Jura, et Mouthe, Doubs, en Franche-Comté), est créée en 1979 sur le modèle de la Vasaloppet (Suède), plus importante épreuve populaire de ski de fond au monde. Elle devient au fil du temps, avec l'aide et l'appui financier des collectivités locales (communes traversées par la course, départements, région), la vitrine identitaire, touristique et sportive régionale, par la construction de la figure du "skieur jurassien", puisqu'un un tiers des inscrits à cette épreuve internationale sont des Francs-Comtois (Christophe Hanus) ;

- l'huître de l'étang de Thau, valorisée comme patrimoine gastronomique lagunaire par l'agritourisme (Pierre Sécolier);

- l'écotourisme et le patrimoine : la demande internationale commençant à préférer le "vert" au "bleu", le tourisme devient un moyen de mise en valeur les zones enclavées du territoire. Madagascar décide de pratiquer l'écotourisme pour protéger les régions rurales des effets du tourisme de masse dans le contexte d'une campagne de promotion "Madagascar Naturellement" (Jean-Claude et Noëline Ramandimbiarison);

- la valorisation du patrimoine culturel et la recherche d'identité nationale à l'île Maurice (Julie Peghini).

5 Personnellement, l'intérêt que j'ai trouvé à cet ouvrage concerne la place dorénavant faite au sport en tant qu'objet du patrimoine, et en tant qu'objet en voie de patrimonialisation. Tous ces menus objets du quotidien sportif (le "petit patrimoine" dont parle Laurent Sébastien Fournier) que les individus ou les associations constituent en reliques d'un passé qui ravive les souvenirs et les émotions. Ce "petit patrimoine" participe à faire vivre les identités, souvent locales, voire et surtout de quartier ou de village. Mais les textes montrent aussi la diversité de ce patrimoine sportif: la Transjurassienne participe désormais de l'identité de ce massif, comme le Tour de France est l'identité de la France, malgré l'internationalisation de ses participants et même de son parcours qui joue avec les frontières des pays limitrophes... Cela ouvre des perspectives intéressantes pour la recherche sur le sport, par le développement d'autres aspects de la socialisation que la pratique sportive elle-même, car tous ces symboles aux dimensions variables (maillots, emblèmes, coupures de presse, stades, photographies, etc.) marquent de manière indélébile les esprits des pratiquants.

Cet ouvrage est donc extrêmement varié au regard de la diversité des exemples rapportés. Il ne peut que retenir l'attention de tous les lecteurs intéressés par les questions relatives au patrimoine. 